Journal for ImmunoTherapy of Cancer

\title{
Human macrophages engineered to secrete a bispecific $T$ cell engager support antigen-dependent $T$ cell responses to glioblastoma
}

\author{
Jennifer L Gardell (1) , , Lisa R Matsumoto, ${ }^{1}$ Harrison Chinn, ${ }^{1}$ Kole R DeGolier, ${ }^{1,2}$ \\ Shannon A Kreuser, ${ }^{1}$ Brooke Prieskorn, ${ }^{1}$ Stephanie Balcaitis, ${ }^{1}$ Amira Davis, ${ }^{1}$ \\ Richard G Ellenbogen, ${ }^{3,4}$ Courtney A Crane $e^{4,5,6}$
}

To cite: Gardell JL, Matsumoto LR, Chinn $\mathrm{H}$, et al. Human macrophages engineered to secrete a bispecific $T$ cell engager support antigen-dependent $\mathrm{T}$ cell responses to glioblastoma. Journal for ImmunoTherapy of Cancer 2020;8:e001202. doi:10.1136/jitc-2020-001202

- Additional material is published online only. To view please visit the journal online (http://dx.doi.org/10.1136/jitc2020-001202).

Accepted 18 September 2020

Check for updates

(C) Author(s) (or their employer(s)) 2020. Re-use permitted under CC BY-NC. No commercial re-use. See rights and permissions. Published by BMJ.

For numbered affiliations see end of article.

\section{Correspondence to}

Dr Courtney A Crane, Discovery and Translational Science, Mozart Therapeutics, Seattle, WA, USA;

ccrane@mozart-tx.com

\section{ABSTRACT}

Background Targeted and effective treatment options are needed for solid tumors, including glioblastoma (GBM), where survival rates with standard treatments are typically less than 2 years from diagnosis. Solid tumors pose many barriers to immunotherapies, including therapy half-life and persistence, tumor penetrance, and targeting. Therapeutics delivered systemically may not traffic to the tumor site. If cellular therapies or drugs are able to access the tumor site, or can be delivered directly within the tumor, treatments may not persist for the duration necessary to reduce or eliminate tumor burden. An approach that allows durable and titratable local therapeutic protein delivery could improve antitumor efficacy while minimizing toxicities or unwanted on-target, off-tissue effects.

Methods In this study, human monocyte-derived macrophages were genetically engineered to secrete a bispecific T cell engager (BiTE) specific to the mutated epidermal growth factor variant III (EGFRvIII) expressed by some GBM tumors. We investigated the ability of lentivirally modified macrophages to secrete a functional BiTE that can bind target tumor antigen and activate $T$ cells. Secreted BiTE protein was assayed in a range of $T$ cell functional assays in vitro and in subcutaneous and intracranial GBM xenograft models. Finally, we tested genetically engineered macrophages (GEMs) secreting BiTE and the proinflammatory cytokine interleukin (IL)-12 to amplify T cell responses in vitro and in vivo.

Results Transduced human macrophages secreted a lentivirally encoded functional EGFRvlll-targeted BiTE protein capable of inducing $T$ cell activation, proliferation, degranulation, and killing of antigen-specific tumor cells. Furthermore, BiTE secreting macrophages reduced early tumor burden in both subcutaneous and intracranial mouse models of GBM, a response which was enhanced using macrophages that were dual transduced to secrete both the BiTE protein and single chain IL-12, preventing tumor growth in an aggressive GBM model.

Conclusions The ability of macrophages to infiltrate and persist in solid tumor tissue could overcome many of the obstacles associated with systemic delivery of immunotherapies. We have found that human GEMs can locally and constitutively express one or more therapeutic proteins, which may help recruit T cells and transform the immunosuppressive tumor microenvironment to better support antitumor immunity.

\section{INTRODUCTION}

Although recent advancements in cancer immunotherapy have shown rapid progress in the elimination of blood cancers, the treatment of solid tumors has progressed much more slowly. This is especially true of glioblastoma (GBM), where therapies such as checkpoint inhibitors and chimeric antigen receptor (CAR) $\mathrm{T}$ cells have not significantly extended patient survival. ${ }^{2}$ Major obstacles to successful immunotherapy for GBM include the restricted recruitment of $\mathrm{T}$ cells to the brain, the immunosuppressive tumor microenvironment (TME), and the low mutagenic burden of most GBM tumors. ${ }^{3}$

Bispecific T cell engagers (BiTEs) are a class of immunotherapeutic proteins designed to facilitate the interactions of $\mathrm{T}$ cells and tumor cells via binding of a tumor-specific antigen on one single chain variable fragment $(\mathrm{scFv})$, and the $\mathrm{CD} 3 \varepsilon$ receptor on $\mathrm{T}$ cells, with the goal of inducing a polyclonal $\mathrm{T}$ cell response to a tumor antigen. Dual engagement of the BiTE with the $\mathrm{T}$ cell and target cell induces $\mathrm{T}$ cell activation and release of lytic granules that result in target cell killing. Therefore, BiTEs, much like CAR T cells, offer a method to activate polyclonal $\mathrm{T}$ cell populations in a non-Major Histocompatibilty Complex (MHC)-restricted manner to kill cancer cells. In contrast to CAR T cells, BiTEs are relatively small, and therefore better able to penetrate solid tumors, where they could engage T cells. BiTE stimulation not only induces killing of tumor cells ${ }^{4}$ but can also reverse the immunosuppressive functions of regulatory $\mathrm{T}$ cells often described in GBM tumors. ${ }^{5}$ 
Indication-specific BiTEs are approved for the treatment of leukemia and are in clinical trials for the treatment of many types of solid tumors. ${ }^{6}$ A BiTE targeted to the mutated epidermal growth factor variant III (EGFRvIII), which is expressed by tumor cells in $30 \%$ of GBM patient tumors, has shown significant promise in preclinical models, ${ }^{7}$ and is currently being tested in patients. ${ }^{8}$ However, BiTEs also have disadvantages, such as suboptimal tissue penetrance, lack of tumor targeted delivery, and short half-lives. ${ }^{9}$ These disadvantages require frequent high-dose systemic administration to achieve efficacy, which can be associated with off-target toxicities. ${ }^{10}$ Reducing treatment frequency and toxicities through sustained local delivery may therefore be of benefit to improving outcomes and quality of life for patients treated with BiTEs.

We have previously reported an engineered macrophage cellular therapy that capitalizes on the natural penetrance and accumulation of macrophages in the GBM TME. ${ }^{11}$ Tumor tissue penetrance and long-term persistence of engineered macrophages in vivo suggest that this platform could alter the TME, allowing recruitment and activation of antitumor immune cells. In this study, we engineered human monocyte-derived macrophages to secrete a BiTE specific to the tumor antigen EGFRvIII, and evaluated the ability of secreted BiTE to trigger $\mathrm{T}$ cell activation and killing of tumor cells in vitro and in vivo. BiTE-secreting genetically engineered macrophages (GEMs) induced $\mathrm{T}$ cell activation and tumor cell killing in an EGFRvIII-dependent fashion. In vivo, EGFRvIII BiTE GEMs suppressed early tumor growth. Dual-transduced GEMs secreting EGFRvIII BiTE and interleukin (IL)-12 similarly activated T cell killing of tumor targets, and were sufficient to prevent tumor GBM growth in vivo. Collectively, our data suggest that GEMs may be used as a stand-alone treatment or as an adjuvant to other immunotherapy approaches. ${ }^{12-16}$

\section{MATERIALS AND METHODS \\ Viruses}

EGFRvIII lentivirus was used to generate EGFRvIIIexpressing U87 cell lines. BiTE, truncated CD19 negative control (CD19t), mCherry, and IL-12 sequences were synthesized by GeneArt (Carlsbad, California, USA) and inserted in epHIV7.2 (11) backbone between NheI and Not1 restriction enzyme sites. This plasmid was transfected into 293T cells along with modified packaging plasmids and a plasmid encoding the Vpx protein as described. ${ }^{11}$ Virions were collected from transfected 293T cell supernatants and concentrated by ultracentrifugation $\left(90 \mathrm{~min}, 24.5 \mathrm{~K} \mathrm{rpm}, 4^{\circ} \mathrm{C}\right)$. Viral titer was determined using the QuickTiter Lentivirus Titer kit (Cell Biolabs).

\section{Cell lines}

293T (ATCC-CRL-1326) and U87 (ATCC-HTB14) cell lines were obtained from ATCC. EGFRvIII eGFP-ffluc Raji and EGFRvIII K562s were a gift from the Jensen lab. EGFRvIII overexpressing U87s were generated by transduction of $1.0 \times 10^{6} \mathrm{U} 87 \mathrm{~s}$ with EGFRvIII-epHIV7.2 and eGFP-ffluc-epHIV7.2 at an multiplicity of infection (MOI) of 1 with $0.025 \mathrm{mg} / \mathrm{mL}$ protamine sulfate (Fresenius Kabi).

\section{Macrophages}

Human monocyte-derived macrophages were generated by CD14 isolation from healthy plasma donor peripheral blood products (BloodWorks NW) using a previously described protocol. ${ }^{11}$ Briefly, CD14+ cells were isolated using an Easy Sep Human CD14 positive selection kit (Stem Cell Technologies) and differentiated with $10 \mathrm{ng} /$ $\mathrm{mL}$ human granulocyte-macrophage stimulating factor (GMCSF) (R\&D Systems) in Roswell Park Memorial Institute (RPMI) (Gibco)/10\% fetal bovine serum (FBS) (Peak Serum). Three days later, $100 \%$ of cytokine and $50 \%$ media were refreshed. On day 6 , cells were trypsinized, counted, and replated $\left(60000\right.$ cells $\left./ \mathrm{cm}^{2}\right)$.

\section{T cells}

$\mathrm{T}$ cells were isolated from macrophage donor-matched peripheral blood mononuclear cells (PBMCs) using the Human CD3 Positive Selection Kit II (Stem Cell Technologies). Cells were plated at stated densities with $30 \mathrm{U} / \mathrm{mL}$ recombinant human IL-2 (rhIL-2) where indicated (R\&D Systems). T cells were stimulated with Dynabeads (Gibco) according to manufacturer's instructions.

\section{Antibodies and cell stains}

Flow cytometry antibodies

Biolegend: CD19-PE (clone HIB19), CD4-Alexa647 (clone OKT4), CD4-BV510 (clone peripheral), CD3BV605 (clone OKT3), CD69-PE (clone FN50), CD69-FITC (clone FN50), CD107a-BV421 (clone H4A3), PD-1-PECy7 (clone EN12.2H7), IFN $\gamma$-BV785 (clone 4S.B3), TNFoAlexa647 (clone Mab-11), CD40L-PEDazzle594 (clone 24-31),

Becton Dickinson: CD3-FITC (clone UCHT-1), CD8PerCPCy5.5 (clone RPA-T8), CD25-APC-H7 (clone M-A251), Granzyme B-A700 (clone GB11), Fc block (Clone: 3070), Fixable Viability Stain 520 (BD Horizon), Live/Dead UV450/50 (Invitrogen), Cell Trace Violet (Invitrogen), His-PE (Miltenyi, clone GG11-8F3.5.1), anti-6X His tag antibody [HIS.H8] (AbCAM, Cat. No. ab18184), IRDye 800CW Goat anti-mouse IgG secondary antibody (Li-COR Biosciences, Cat. No. 926-32210).

\section{Woodchuck hepatitis virus post-transcriptional response element} (WPRE) assay

Transduced macrophage (day 7 post-transduction) genomic DNA was isolated, according to the manufacturer's recommended protocol (Qiagen), concentrated to $50 \mathrm{ng} / \mu \mathrm{L}$, and quantitative PCR (qPCR) was performed. Further, 10-point standard curves were generated using epHIV7 (1 WPRE construct/molecule) and pCMV6-AC (1 albumin construct/molecule). Copy number/cell was determined by normalizing WPRE starting quantity (sq) 
to albumin sq using the following equation: (WPRE sq/ albumin $\mathrm{sq} \times 2$ ).

\section{CD19 truncated marker staining}

Macrophages transduced with 125-750 LP/cell BiTEencoding lentivirus, dual transduced with BiTE (750 LP/ cell) and IL-12 (250 LP/cell) lentiviruses, or untransduced were harvested on day seven post-transduction with TrypLE Express. Macrophages were incubated with human Fc block $(50 \mu \mathrm{g} / \mathrm{mL})$, stained with a live/dead dye and anti-CD19 antibody, fixed with $2 \%$ paraformaldehyde, and analyzed by flow cytometry.

\section{Western blot}

293T supernatant was collected 4 days post-transfection and then purified according to recommended protocol with protein $\mathrm{L}$ magnetic beads (Pierce) or a nickel column generated from Nickel (Ni) Sepharose 6 Fast Flow beads in HisTrap FF columns (GE Healthcare). Purified proteins were run on an Sodium Dodecyl Sulfate Polyacrilamide Gel Electrophoresis (SDS-PAGE) gel and then transferred to a Millipore membrane. Following transfer, the membrane was blocked with Odyssey blocking buffer (LI-COR) and probed with anti-His antibody (Abcam HIS-H8, ab18184, 1:1000), followed by an anti-mouse secondary antibody (Li-COR, 926-32210, 1:10000). The membrane was imaged and analyzed on an Odyssey CLx Infrared Imaging System.

\section{ELISA}

293T cells were transfected with $2.5 \mu \mathrm{g}$ plasmid DNA encoding the BiTE lentivirus with GMCSF and H7 secretion sequences according to the Mirus Bio TransIT-LT1 Transfection protocol and supernatant was collected 3 days later. BiTE-transduced macrophage supernatant was collected from $3 \times 10^{6}$ transduced macrophages plated in $10 \mathrm{~cm}$ dish (days 7-14). Supernatant was concentrated using a $10 \mathrm{~mL}$ spin column $(4000 \times \mathrm{g}$ for $20 \mathrm{~min})$ and purified using Ni Sepharose 6 Fast Flow (GE Healthcare) beads followed by protein L magnetic beads (Pierce). Then, $50 \mu \mathrm{L}$ was added to the His ELISA according to manufacturer's protocol.

\section{EGFRvIll binding assay}

Unconcentrated supernatant from transfected 293T (day 3) or transduced macrophages (day 7) was added to $1.0 \times 10^{6}$ EGFRvIII-overexpressing K562 cells for $20 \mathrm{~min}$. Cells were subsequently stained with anti-His PE antibody (Miltenyi, clone GG11-8F3.5.1) and analyzed using flow cytometry.

\section{Gene expression analysis}

$5.0 \times 10^{5}$ GMCSF-differentiated macrophages were transduced and cultured with $2.0 \times 10^{5}$ EGFRvIII-expressing U87s and $3.0 \times 10^{6} \mathrm{~T}$ cells isolated from autologous PBMCs. Three days later, $\mathrm{T}$ cells in suspension were collected and RNA prepared using the RNeasy Mini Kit (Qiagen). Further, 25 ng of RNA was analyzed using the human immunology v2 panel (NanoString). Threshold values were defined as two times the average background of negative controls, and gene expression was normalized to internal housekeeping genes. Secreted proteins were quantified using the Bio-Plex Pro Human Immunotherapy Panel, 20 plex (BioRad) and analyzed using the Bio-Plex Manager Software.

\section{T cell coculture assays}

Supernatant from $5.0 \times 10^{5}$ transduced macrophages or $2 \mathrm{~mL}$ transfected 293T cells were cultured with T cells and EGFRvIII-K562 or U87 target cells (3-4 days). Cells were stained for CD3, CD4, CD8, CD25, CD69, and PD-1 and Live/Dead. For degranulation assays, T cells were added to transduced macrophages (day 6 post-transduction) for 2 days prior to the addition of target cells, FcR blocking antibody, and CD107a antibody for 6 hours. For proliferation assays, $T$ cells were labeled using the CellTrace Cell Proliferation Kit (Invitrogen) and incubated for 6 days, with introduction of $2.0 \times 10^{5}$ new targets on day 3 . For intracellular staining, brefeldin A was added 5 hours prior to harvesting cells and staining. All samples were run on a BD LSR Fortessa flow cytometer using FACS DIVA software and analyzed with FlowJo V.10.

\section{Phagocytosis assays \\ Bead assay}

GEMs were incubated on day 7 post-transduction with $500 \mu \mathrm{L}$ resuspended pHrodo RED particles (Invitrogen) for $90 \mathrm{~min}$ at $37^{\circ} \mathrm{C}$. Following incubation, macrophages were lifted with TrypLE and analyzed via flow cytometry.

\section{Incucyte}

Macrophages were transduced with mCherry lentivirus at $500 \mathrm{LP} /$ cell in combination with CD19t (750 LP/cell), BiTE (750 LP/cell), or BiTE (750 LP/cell) and IL-12 (250 LP/cell) lentivirus. Six days post-transduction, GEMs were replated at 62500 cells/well. The following day, 20833 EGFRvIII eGFP-ffluc Raji target cells were added and the plate was live imaged every $10 \mathrm{~min}$ for 24 hours using the Incucyte live cell imager (Essen Biosciences, Ann Arbor, Michigan, USA). Green Fluorescent Protein (GFP) counts were determined over time.

\section{Chromium (Cr-51) release assay}

GMCSF-differentiated macrophages were plated at $1.5 \times 10^{4} /$ well. Twenty-fourhours later, macrophages were transduced with $750 \mathrm{LP} /$ cell BiTE lentivirus with or without IL-12 lentivirus (250 LP/cell), IL-12 alone (250 LP/cell) or CD19t control lentivirus (750 LP/ cell). Autologous PBMCs were used for CD3 isolation or plated overnight in IL-2 before addition of PBMCs or $\mathrm{T}$ cells to macrophages or $1 \mathrm{ng}$ control purified BiTE protein, followed by addition of $5.0 \times 10^{3}$ Cr51-labeled EGFRvIII-U87 target cells for 18 hours. Supernatants were harvested onto a filter, read on a TopCount, and percent lysis determined. 


\section{IL-12 Bioplex}

Supernatant from $5.0 \times 10^{5} \mathrm{BiTE} / \mathrm{IL}-12$ or IL-12-transduced macrophages was collected 7 days after transduction and analyzed using the IL-12 Bioplex protocol (BioRad).

\section{Immunohistochemistry of mouse tumor samples}

Formalin-fixed paraffin-embedded tumor sections were deparaffinized and pretreated with DIVA decloaking buffer (Biocare medical) at $125^{\circ} \mathrm{C}$. Sections were blocked for 1 hour in $0.2 \%$ Bovine Serum Albumin (BSA) $+2 \%$ normal goat serum (Jackson Immuno). Sections were stained with rat anti-human CD3 (BioRad, clone CD3-12, \#MCA1477) at 1:100 and rabbit anti-human CD8 (Abcam, polyclonal,\#ab4055) diluted 1:100 in blocking buffer for 1 hour, $25^{\circ} \mathrm{C}$. Sections were stained with goat anti-rat AlexaFluor 488 (Thermo, \#A11006) and goat anti-rabbit AlexaFluor 568 (Thermo, \#A11011) each diluted 1:500 in blocking buffer for 1 hour, $25^{\circ} \mathrm{C}$. Sections were then stained for $10 \mathrm{~min}$ in Hoechst dye (Thermo, \#H3570) at $2 \mu \mathrm{g} / \mathrm{mL}$ in $\mathrm{dd}_{2} \mathrm{O}$, and coverslipped with Prolong Gold (Thermo \#P36935). Sections were imaged at $\times 40$ (Plan apo, NA 0.95) on a Nuance Multispectral camera (Akoya Biosciences) with a Nikon Eclipse $\mathrm{Ci}$ microscope and images were analyzed with InForm Tissue Analysis software, V.2.4 (Akoya Biosciences).

\section{In vivo subcutaneous models}

$1.0 \times 10^{6}$ eGFP-ffluc EGFRvIII U87s cells in a $50 / 50 \%$ matrigel/cells in $100 \mu \mathrm{L}$ Phosphate Buffered Saline (PBS) were injected subcutaneously into the right flank of NSG mice (Jackson Laboratory). Eighteen days later, $1.0 \times 10^{6}$ GEMs were injected intratumorally, followed by intravenous injection of $1.0 \times 10^{7}$ Dynabead activated T cells. Positive control animals received five daily intratumoral injections of $1 \mu \mathrm{g}$ purified BiTE protein and $12 \mathrm{ng}$ rhIL-12 starting on day 18 . For the BiTE and IL-12 GEM combination study, $1.0 \times 10^{6}$ eGFP-ffluc EGFRvIII U87s and $5 \times 10^{5}$ GEMs with or without $2.5 \times 10^{5}$ IL-12 GEMs were injected subcutaneously into the right flank of NSG mice. Seven days later, mice received an intravenous injection of $1 \times 10^{7}$ Dynabead activated $T$ cells that had been rested 5 days in $30 \mathrm{U} / \mathrm{mL}$ recombinant human IL-2. A single injection of intratumoral human purified BiTE protein $(10 \mathrm{ng})$ or BiTE protein in combination with recombinant human IL-12 (12 ng) on the day of T cell injection was given as a control. Tumor growth was measured using bioluminescence and tumor volume twice weekly. For luminescence measurements, mice were anesthetized with isoflurane and injected subcutaneous with D-luciferin. Fifteen minutes post-luciferin injection, mice were imaged on an Xenogen IVIS In Vivo Imaging System. Tumor volume was calculated using the following formula: volume $=\left(\right.$ width $^{2} \times$ length $\left./ 2\right)$. Mice were euthanized when tumor size reached $2000 \mathrm{~mm}^{3}$.

\section{In vivo intracranial model}

Intracranial injection of eGFP-ffluc EGFRvIII U87s was performed as previously described. ${ }^{17}$ Briefly, $2.0 \times 10^{5}$
eGFP-ffluc EGFRvIII U87s in $2 \mu \mathrm{L}$ of PBS were injected intracranially ( $2 \mathrm{~mm}$ lateral, 0.5 anterior to bregma and $2.5 \mathrm{~mm}$ deep from dura). Six days later, $3.0 \times 10^{5} / 6 \mu \mathrm{L}$ GEMs were injected into the tumor. $3.0 \times 10^{5} \mathrm{~T}$ cells were injected intratumorally 3 days later, and tumor burden was measured by bioluminescence three times weekly.

\section{RESULTS}

In approximately $30 \%$ of patients with GBM, tumor cells express a mutated form of EGFR with a constitutively active tyrosine kinase, known as EGFRvIII. ${ }^{8}$ We created a macrophage-specific lentiviral vector encoding a BiTE that binds EGFRvIII (806 single scFv) and the T cellengaging anti-CD3 OKT3 scFv, and modified the design to enhance production using short linker as described ${ }^{6}$ and added a histidine (His) tag (online supplemental figure 1A). To validate the EGFRvIII BiTE construct function, supernatant was harvested from transfected 293T cells and cultured with T cells. 293T BiTE supernatant increased expression of the activation markers CD25 (online supplemental figure 1B) and CD69 (online supplemental figure 1C) on CD4 and CD8 T cells cultured with EGFRvIII-expressing K562 target cells. Supernatants from transfected 293T cells and GEMs were purified using a nickel column $(\mathrm{Ni}+)$ or magnetic protein $\mathrm{L}$ beads $(\mathrm{L}+)$ after which BiTE protein was detected using an anti-His antibody (online supplemental figure 1D), validating that the BiTE construct encodes a functional secreted protein.

BiTE GEMs were generated by transducing human monocyte-derived macrophages with BiTE-encoding epHIV7.2 lentivirus. ${ }^{11}$ As macrophages do not express endogenous CD19, a truncated, non-signaling CD19 $(\mathrm{CD} 19 \mathrm{t})^{18}$ was encoded downstream of the BiTE sequence to measure transduction efficiency (online supplemental figure 1A). Flow cytometry showed that CD19t positive macrophages increased with escalating doses of lentivirus (figure 1A). Consistent with this observation, analysis of viral integration events, performed by quantifying WPRE copy numbers, increased with lentiviral concentrations $(n=3$ donors, figure 1B). Based on these data, macrophages were transduced using 750 lentiviral particles (LPs) per cell for subsequent experiments (figure 1A, online supplemental figure $2 \mathrm{~A}$ ).

To ensure that no post-translational events prevent BiTE secretion by GEMs, we tested supernatant after transduction relative to the negative control macrophages transduced with lentivirus encoding only CD19t (CD19t GEMs). Although we did not detect BiTE protein by western blot (online supplemental figure 1D), it was detectable in BiTE GEM supernatant relative to CD19t GEMs using a competitive binding ELISA over 7 days (online supplemental figure 2B). Concentrated GEM supernatants were tested for BiTE secretion, contained an average of $63.4 \mathrm{ng} / \mathrm{mL}$ (figure 1C), a 16.4-fold increase over control CD19t GEMs. To determine if there was a correlation between integration events and BiTE protein concentration, we ran a WPRE assay and a sample-matched ELISA 
A

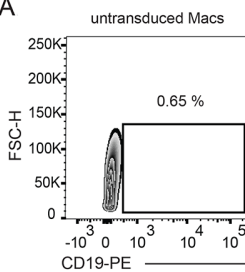

500 LP/cel

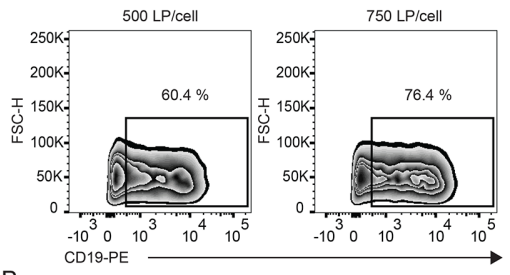

B

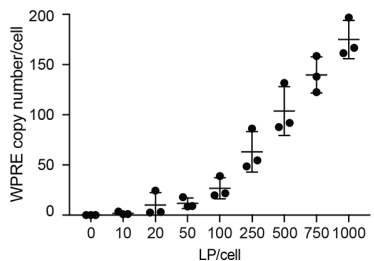

C

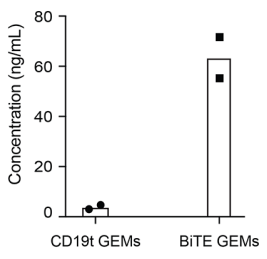

D

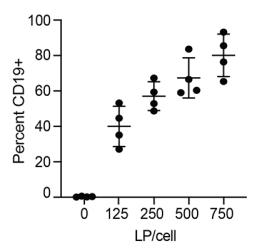

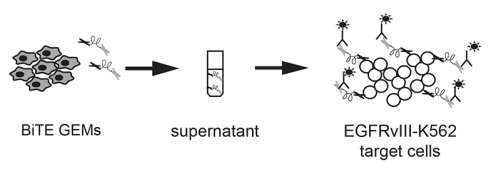

E

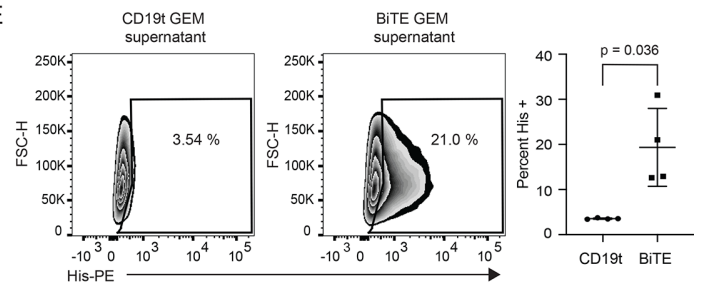

Figure 1 Bispecific T cell engager (BiTE) lentivirus integration results in detectable amounts of secreted BiTE protein by genetically engineered macrophages (GEMs). (A) $5 \times 10^{5}$ macrophages were transduced at increasing concentrations of BiTE lentivirus per macrophage and the percentage of positively transduced macrophages was detected by the truncated CD19 (CD19t) marker. Representative dot plots of CD19 positive cells for each condition are shown as determined by a Phycoerythrin (PE)-labeled CD19 antibody with untransduced macrophages as a negative control. The graph shows quantification of CD19 staining for each condition for four independent donors. (B) BiTE GEMs were generated from three independent donors with increasing doses of lentiviral particles (LP) per macrophage. The graph shows integration events as determined from day 7 post-transduction genomic DNA for each dose tested. (C) Supernatant accumulated for 14 days following transduction of $3 \times 10^{6} \mathrm{BiTE}$ or CD19t control GEMs at $750 \mathrm{LP} / \mathrm{cell}$ was concentrated and BiTE protein was purified with protein L magnetic beads. Concentration of BiTE in the supernatant was determined using a competitive inhibition ELISA and is shown for two independent donors. (D) A representative diagram of the BiTE binding assay for detection of BiTE protein is shown. Supernatant was harvested 7 days following transduction with the lentivirus encoding the BiTE protein and added to epidermal growth factor receptor variant III (EGFRVIII)-expressing K562s. BiTE binding to target antigen was detected with a PE-labeled anti-His antibody by flow cytometry. (E) Representative dot plot showing His staining on EGFRvIll-K562s following incubation with BiTE GEM or CD19t control GEM supernatant. Quantification of percentage of His positive cells for BiTE and CD19t control GEMs is shown for four independent donors in the graph on the right.

( $\mathrm{n}=9$ donors). When transduced at $750 \mathrm{LPs} /$ cell, there was a range of integration frequency from 150 to almost 500 copies/cell, which did not predict the secreted BiTE protein concentration (online supplemental figure $2 \mathrm{C}$ ).

To test if lentivirally encoded BiTE secreted by macrophages bound the intended EGFRvIII tumor antigen, we cultured BiTE GEM supernatants with EGFRvIIIoverexpressing K562 cells and stained cells using a fluorescently conjugated anti-His antibody (figure 1D). Relative to control GEM supernatants, a significant percentage of target cells bound the His-tagged BiTE protein (figure $1 \mathrm{E}, 19.35 \%$ vs $3.49 \%$, $\mathrm{p}=0.036$ ), indicating antigen-specific binding.

To optimize engineered BiTE secretion by GEMs, we compared a granulocyte-macrophage stimulating factor (GMCSF) secretion sequence and the antibody-specific heavy chain signal peptide 7 (H7) sequence, which is derived from the human immunoglobulin heavy chain sequence, and enhances antibody secretion in Chinese Hamster Ovary (CHO) cells. ${ }^{19}$ Although there was not a significant difference in BiTE protein secreted by transfected 293T cells (online supplemental figure 3A), the $\mathrm{H} 7$ secretion sequence BiTE GEMs induced consistently greater $\mathrm{T}$ cell activation than GEMs with the GMCSF secretion sequence BiTE (online supplemental figure 3B,C). The H7 secretion sequence was therefore incorporated into the lentiviral vector for subsequent experiments.

One advantage of using macrophages for protein delivery is their potential to provide essential costimulatory signals to tumor-infiltrating $\mathrm{T}$ cells. Following confirmation of EGFRvIII binding by the GEM-secreted BiTE, we tested the effects of GEMs on T cell activation. BiTE GEMs significantly increased expression of the activation markers CD25 (figure 2A) and CD69 (figure 2B) on the surfaces of CD4 (CD25, p<0.0001, CD69, p=0.0086) and CD8 (CD25, p=0.0233, CD69, $\mathrm{p}=0.0019) \mathrm{T}$ cells in the presence of antigen-expressing GBM tumor cell targets (U87+) relative to control CD19t GEMs. T cells also significantly increased the expression of CD40L (AVG CD4/ CD8) (figure 2C, $\mathrm{CD} 4 \mathrm{p}=0.0475, \mathrm{CD} 8 \mathrm{p}=0.0067$ ), interferon $\gamma(\mathrm{IFN} \gamma$ ) (figure 2D, CD4 p=0.0001, CD8 p=0.0001), 

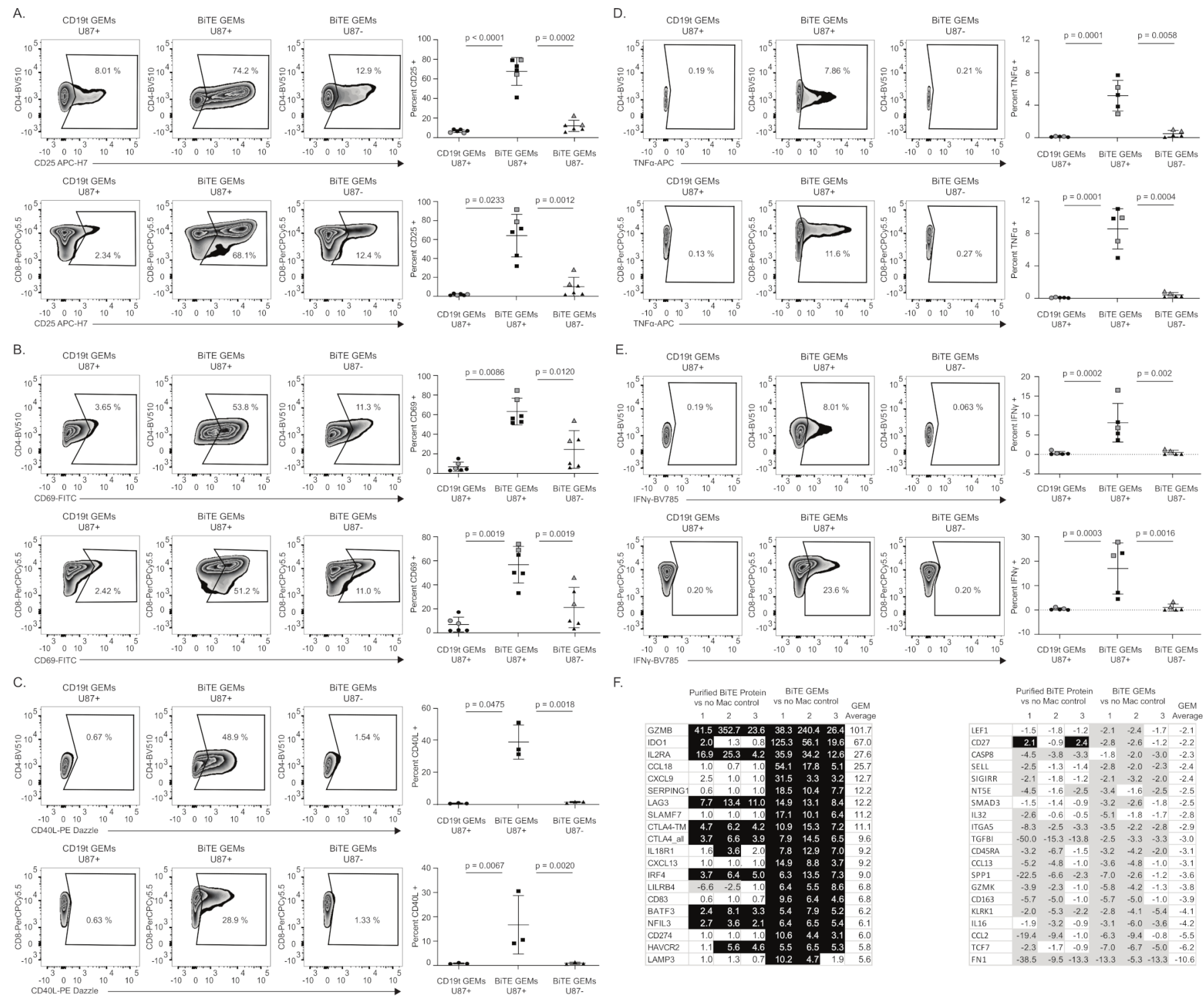

Figure 2 Bispecific T cell engager (BiTE) genetically engineered macrophages (GEMs) induce T cell activation and cytokine production. Representative dot plots of CD25 (A) or CD69 (B) activation markers and CD40L (C) on CD4 or CD8 T cells following a 3-day incubation with $5 \times 10^{5} \mathrm{BiTE}$ or truncated CD19 (CD19t) control GEMs and epidermal growth factor receptor variant III (EGFRvIII) U87 (U87+) target cells or U87 control (U87-) control cells. (D, E) Brefeldin A was added to T cell:GEM:target cell cultures for 5 hours prior to harvesting the cells for intracellular cytokines. Representative dot plots of tumor necrosis factor $\alpha(T N F \alpha)(D)$ or interferon $\gamma$ (IFN $\gamma$ ) (E) cytokine production by CD4 or CD8 T cells following a 3-day incubation with BiTE or CD19t control GEMs and EGFRvIlI U87 target or U87 control cells. For each activation marker and cytokine, quantification of the percentage positive CD4 or CD8 T cells under each condition is shown in the graph on the right. Each symbol represents results from an independent donor. Each condition was run in duplicate, except as indicated in gray. (A-E) Cells were plated at a ratio of 25T cells:3 GEMs:1 target cell. Bars on graphs represent mean and SD for each marker. P values were determined using a paired ratio, two-tailed t-test. (F) $3 \times 10^{6}$ autologous T cells were incubated with $5 \times 10^{5}$ BiTE GEMs or 10 ng purified BiTE protein and $2 \times 10^{5}$ EGFRvIll U87 target cells for 3 days. On day 3 , RNA was isolated from the indicated cocultures and $25 \mathrm{ng}$ of RNA was hybridized and run on human immunology Nanostring v2 panel. The graph shows the top 20 upregulated (black) or downregulated (gray) genes for the T cells cultured with target cells and BiTE GEMs or purified BiTE protein in comparison to cocultures in the absence of macrophages. Results are shown for three independent donors as listed above the chart, donors 1-3 (1-3).

and tumor necrosis factor $\alpha(\mathrm{TNF} \alpha)$ (figure 2E, CD4 $\mathrm{p}=0.0002$, CD $8 \mathrm{p}=0.0003$ ). Within 5 hours, increases in $\mathrm{T}$ cell cytokine production were measurable relative to controls (online supplemental figure 4A,B). Consistent with prolonged activation, $\mathrm{T}$ cells increased programmed death-1 (PD-1) in coculture (online supplemental figure 4C).
To compare the effects of BiTE GEMs relative to BiTE protein alone, we tested gene expression in T cells incubated with EGFRvIII-U87 target cells and either BiTE GEMs or equivalent concentrations of purified BiTE protein. BiTE GEMs increased genes associated with $\mathrm{T}$ cell activation and IFN $\gamma$ signaling (SLAMF7, IL2RA, BATF3, IRF4), T cell recruitment (CCL18, CXCL9, 
CXCL13), and killing (GZMB, LAMP3) relative to $\mathrm{T}$ cells incubated with purified BiTE protein (figure $2 \mathrm{~F}$ ). Gene ontogeny analysis indicated that the 50 genes with the greatest increase in expression were involved in cell responses to stimuli, cell communication, and signaling (online supplemental figure 4D).

Consistent with increased cytokine production detected by flow (figure 2D and E), exposure to BiTE GEMs significantly increased secreted TNF $\alpha(\mathrm{p}=0.0059)$ and IFN $\gamma(p=0.0052)$ in coculture supernatants (figure 3A). BiTE GEMs also induced cytokines necessary for $\mathrm{T}$ cell survival (figure 3B), such as IL-2, IL-7, and IL-15, and those associated with different helper $\mathrm{T}$ cell subsets (figure $3 \mathrm{C}$ ). In addition to $\mathrm{T}$ cell-specific proinflammatory cytokines, interferon-inducible chemokine (IP-10, $\mathrm{p}=0.0018)$ and cytokines associated with an inflammatory immune response, IL-8 ( $\mathrm{p}=0.0032)$ and IL-18 $(\mathrm{p}=0.0049)$ were also significantly increased (figure 3D). GEMs cocultured with autologous $\mathrm{T}$ cells also retained their ability to phagocytose both beads using flow cytometry (figure 3E, left) and tumor cells using longitudinal Incucyte imaging (figure 3E, right), which was independent of lentiviral transduction, suggesting that GEMs may both contribute to tumor cell clearance and present tumor antigens to $\mathrm{T}$ cells following phagocytosis. ${ }^{20} 21$

Analysis of $\mathrm{T}$ cell gene expression ( $\mathrm{n}=3$ donors, performed in triplicate) showed that during coculture, BiTE GEMs induced genes important for $\mathrm{T}$ cell survival (IL2RA and IL2RB), memory development (PRDM1), cytokine signaling (CISH, IFNG, TNFAIP3, TNFRSF4, TNFRS9, IRF4, and IRF1), CTL response (GZMB and LAMP3), costimulatory molecules (ICOS and CD40), and antigen exposure (CTLA4, SLAMF, LTA, and LAG3) (figure 3F). TGFB1, which promotes tumor growth and suppresses antitumor immunity in glioma, was one of the most downregulated genes (figure $3 \mathrm{~F}$ ).$^{22}$ This gene signature was associated with increased antigen-specific proliferation of CD4 and CD8 T cells (figure 3G), indicating the potential for BiTE GEMs to amplify proinflammatory immune responses by expanding antitumor $\mathrm{T}$ cells.

To measure the impact of BiTE GEMs on T cell cytotoxicity, we tested T cell CD107a and Granzyme B degranulation as well as target lysis. CD4 and CD8 T cells increased CD107a (figure 4A, CD4 p=0.0039, CD8 p=0.0026) and Granzyme B (figure 4B) degranulation in the presence of EGFRvIII tumor targets. Both CD4 T cells (figure 4B, top) and CD8 (figure 4B, bottom) were over $90 \%$ Granzyme B positive when incubated with autologous BiTE GEMs in the presence of EGFRvIII-U87s, representing a 55.25-fold increase for CD4 T cells $(\mathrm{p}=0.003)$ and a 2.19-fold increase for CD8 T cells $(\mathrm{p}=0.0768)$ relative to control cells. Chromium release assays showed that in comparison to $\mathrm{CD} 19 \mathrm{t}$ control GEMs, BiTE GEMs induced significantly more autologous $\mathrm{T}$ cell (figure 4C, $\mathrm{p}=0.005$ ), and PBMC lysis (figure 4E, $\mathrm{p}=0.009$ ) of EGFRvIII-expressing U87 (U87+) cells. The highest concentration of $\mathrm{T}$ cell in the culture (25 T cells:3 GEM:1 U87 target), achieved equivalent lysis of target cells as the positive control purified BiTE protein (figure 4D), without the need to increase the number of GEMs in the coculture (figure $4 \mathrm{C}$ and $\mathrm{E} \mathrm{n}=3$ donors, performed in triplicate). Neither GEMs themselves, nor the immunosuppressive factors secreted by U87 cells, such as TGF $\beta$, impaired antigen-specific killing.

We tested human BiTE GEMs in a subcutaneous (figure 5A-C, online supplemental figure 5A) xenograft mouse model by injecting BiTE GEMs into established EGFRvIII and luciferase-expressing U87 tumors, followed by intravenous injection of purified T cells, and measuring longitudinal bioluminescence. BiTE GEMs delayed tumor growth in early stages when compared with CD19t control GEMs (figure 5C, $\mathrm{p}=0.0815$ ). This trend was consistent, but not as durable, when $\mathrm{T}$ cells were locally injected into an established intracranial model (figure 5D-F, online supplemental figure 5B,C), although the presence of GEMs secreting BiTE delayed tumor growth relative to mice injected with $\mathrm{T}$ cells only (online supplemental figure 5C). In both models, tumors rebounded at later time points, resulting in no significant extension of survival in either system (online supplemental figure $5 \mathrm{~A}, \mathrm{~B})$. In the subcutaneous tumor model, $20 \%$ of the mice from the BiTE GEM group survived longer than mice in the CD19t control GEM treatment group (online supplemental figure 5A). Histological analysis of tumor sections showed enhanced recruitment of CD3+ CD8+ and CD3+ CD8- $\mathrm{T}$ cells to the tumor following BiTE GEM injection, but not CD19t GEM injection (figure 5G). These findings are consistent with previous works showing that BiTE as a monotherapy has only modest efficacy in mouse tumor models. ${ }^{1323}$

Because IL-12 is a well-known modulator of the TME, ${ }^{24}$ enhancing $\mathrm{T}$ and natural killer (NK) cell cytotoxicity, $\mathrm{T}$ cell differentiation to $\mathrm{T}$ helper 1 cells, and IFN $\gamma$ production, we tested a combination of BiTE GEMs and macrophages engineered to produce IL-12 in vitro. Macrophages were transduced with two separate lentiviral constructs that encoded single chain bioactive IL-12 $2^{25}$ and EGFRvIII BiTE (IL-12/BiTE GEMs). Both constructs include the $\mathrm{CD} 19 \mathrm{t}$ sequence as marker of transduction. Dual transduced cells were $81.4 \% \mathrm{CD} 19$ positive in comparison to the singly transduced BiTE cells $(78.2 \%$ positive), a slight increase that was consistent in four donors (online supplemental figure 6A). Supernatant from dual transduced cells was also tested in comparison to supernatant from BiTE GEMs in the EGFRvIII binding assay as described in figure 1D. Dual transduction modestly decreased secreted BiTE protein binding to target cells (23\% vs $30.9 \%$ ) (online supplemental figure $6 \mathrm{~B})$, as well as the concentration of IL-12 in the supernatant (online supplemental figure 6C). BiTE secretion had the greatest impact on genes involved in $\mathrm{T}$ cell activation, with few genes modulated in $\mathrm{T}$ cells after culture with GEMs secreting only IL-12 relative to CD19t expressing GEMs (figure 6A). The presence of macrophages and the process of transduction, independent of lentiviral vector also supported $\mathrm{T}$ cell activation (online supplemental figure $6 \mathrm{D}, \mathrm{E}$ ), indicating the proinflammatory state of ex 

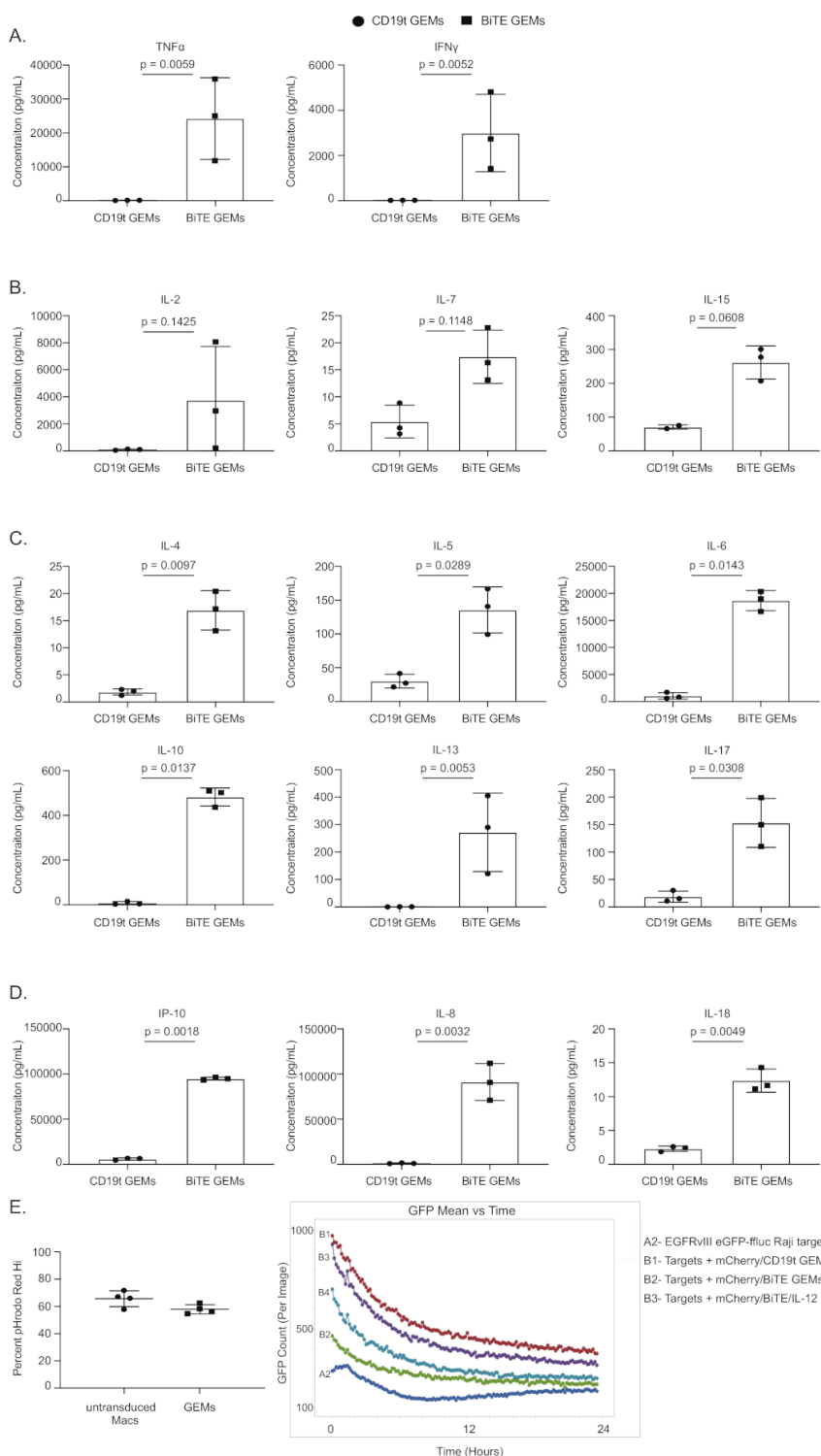
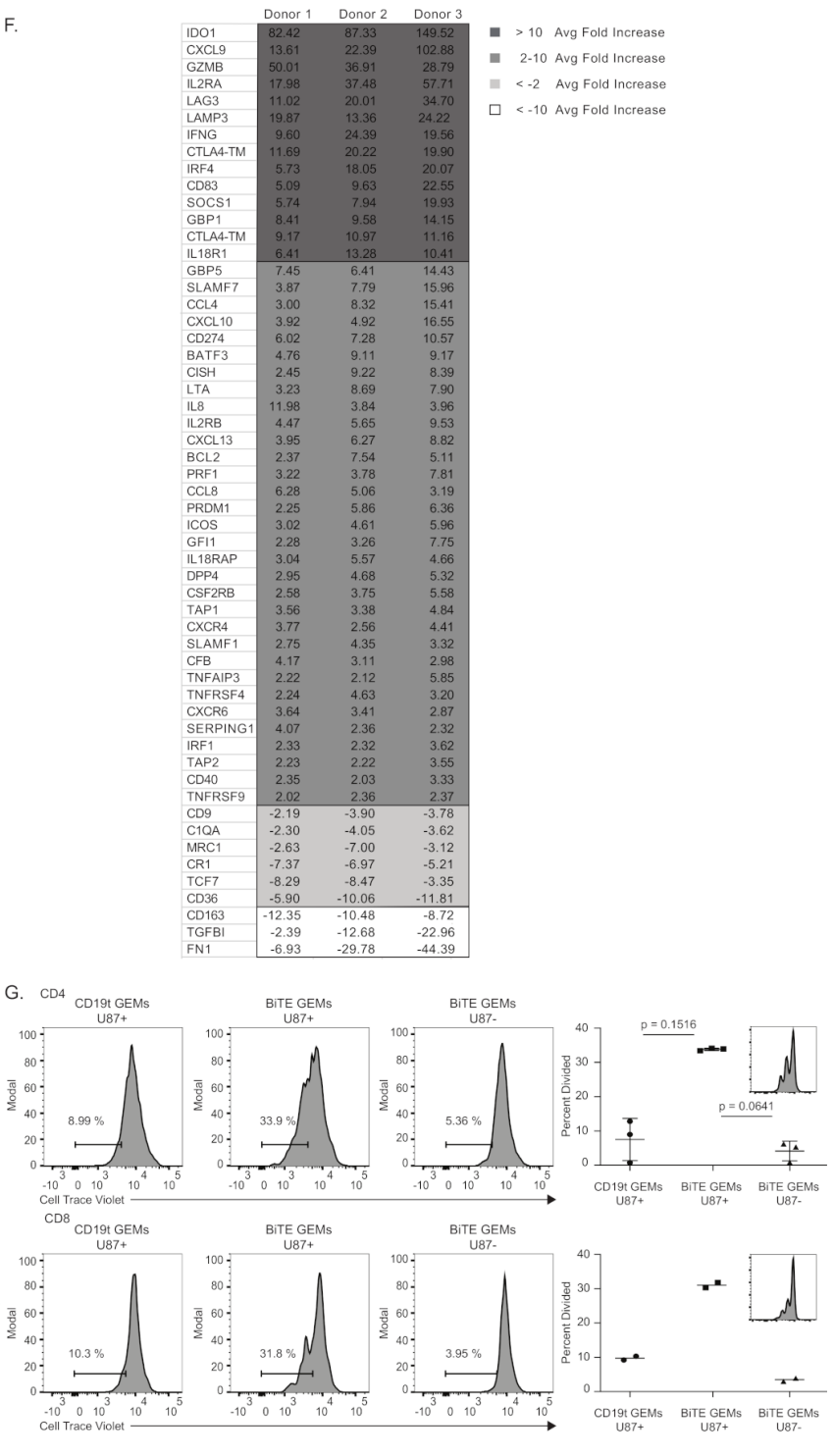

Figure 3 T cell proinflammatory response and proliferation in the presence of bispecific T cell engager (BiTE) genetically engineered macrophages (GEMs). (A-D) Cytokine concentrations detected in the supernatant following the 3-day coculture of $3 \times 10^{6}$ autologous T cells incubated with $5 \times 10^{5} \mathrm{BiTE}$ or CD19t control GEMs and $2 \times 10^{5}$ epidermal growth factor receptor variant III (EGFRvIII) target cells. The graphs depict concentration detected for each analyte in pg/mL for truncated CD19 (CD19t) GEMs (circles) or BiTE GEMs (squares) cultures for three independent donors, run in duplicate. P values list on the graphs were determined using a paired ratio, two-tailed $t$ test. (E) Phagocytosis by GEMs as determined by flow cytometry with pHrodo RED-labeled particles (left) or by incucyte with EGFRvlll eGFP-ffluc Raji tumor target cells and mCherry-labeled GEMs (right). The graph on the left displays percent of pHrodo RED particle Hi positive untransduced macrophages or GEMs following a 90 min coculture with particles. GEMs and untransduced macrophages were differentially labeled with CTV and percent pHrodo RED Hi was determined by flow cytometer, $\mathrm{n}=4$. For the incucyte assay, GEMs were dual transduced with a mCherry lentivirus for visualization and incubated with GFP EGFRvll Raji target cells at a 3:1 ratio and live images were captured every 10 min for 24 hours to determine GFP counts over time. Four live images were taken per condition per time point. (F) Changes in gene expression of $3 \times 10^{6} \mathrm{~T}$ cells incubated with $5 \times 10^{5} \mathrm{BiTE}$ gems over 3 days in the presence of $2 \times 10^{5}$ EGFRvill U 87 target cells relative to cocultures containing truncated CD19 (CD19t) control GEMs. Genes displayed were consistently upregulated or downregulated across three donors over twofold that of the CD19t GEM condition. The scale to the right of the graph shows the key for sorting of the results. Genes with an average fold increase greater than 10 across three donors are shown in dark gray and those with a fold decrease greater than negative 10 are shown in white. (G) Proliferation was measured by dilution of $1 \times 10^{6}$ cell trace violet-labeled CD4 or CD8 T cells following a 6 -day incubation with $5 \times 10^{5} \mathrm{BiTE}$ or CD19t control GEMs and $2 \times 10^{5}$ EGFRvIII U87 target cells with addition of $2 \times 10^{5}$ new U87+ targets on day 3 . Gating for the percentage of divided T cell population is set based on the undiluted, cell trace violet high-expressing cells peak of $T$ cells incubated with control target U87s. Representative histograms for CD4 or CD8 T cells are shown and quantified from two or three independent donors in the graphs to the left. Bars represent mean percentage of dividing T cells with error bars displaying SD. Proliferation on optimal CD3 CD28 Dynabeads are shown for a reference in the corner of the graph. $P$ values list on the graphs were determined using a paired ratio, two-tailed t-test. 


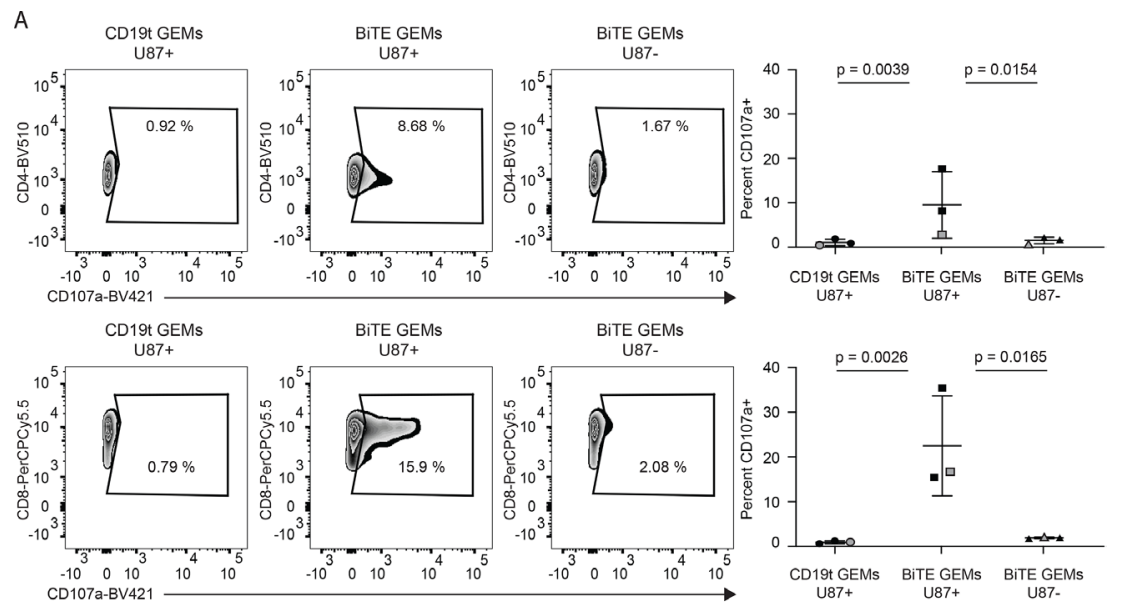

B
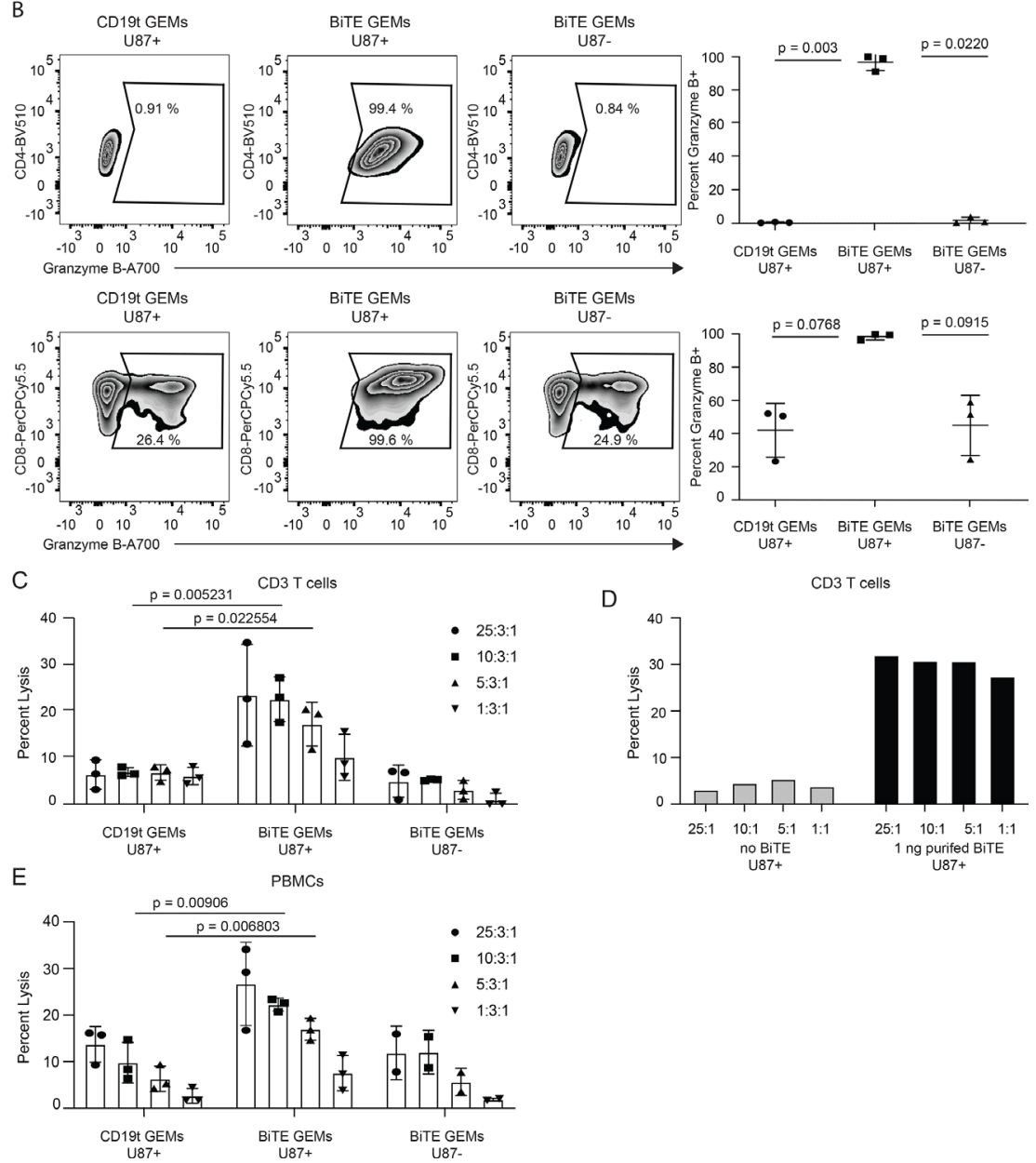

Figure 4 Bispecific T cell engager (BiTE) genetically engineered macrophages (GEMs) induce T cell degranulation and killing of epidermal growth factor variant III (EGFRvIII)-expressing glioma cells. Percentage CD107a (A) or Granzyme B (B) positive CD4 or CD8 T cells following incubation with GEMs and EGFRvIll U87 (U87+) target cells or U87 controls (U87-). (A) Purified CD3 T cells were incubated for 48 hours with BiTE GEMs prior to the addition of U87+ or U87- target cells for 5 hours. (B) T cells, BiTE GEMs or truncated CD19 (CD19t) control, and U87+ or U87- target cells were incubated for 3 days at a ratio of 25:3:1. Percentage of CD4 or CD8 T cells positive for Granzyme B following a 5-hour incubation with Brefeldin A is shown in representative dot plots. (A, B) Individual symbols in the graph to the right are representative of three independent donors, run in duplicate, and bars represent mean and SD per condition. Samples not run in duplicate are indicated with gray symbols. Purified CD3 T cells $(C, D)$ or peripheral blood mononuclear cells (PBMCs) (E) were incubated at specified ratios with (C) BiTE or CD19t control GEMs or (D) purified protein in a 96-well plate. Chromium pulsed target cells were added for 18 hours following initial T cell and GEM incubation and percent lysis was determined based on chromium released into the supernatant during incubation relative to controls. (C, E) Each symbol or bar (D) represents results from an independent donor with all ratios indicated run in triplicate. Ratios listed specify T cell:GEM:target cell. P values are listed for BiTE compared with the CD19t control GEM condition at 10:3:1 and 5:3:1 ratios as determined by a paired t-test. 
A

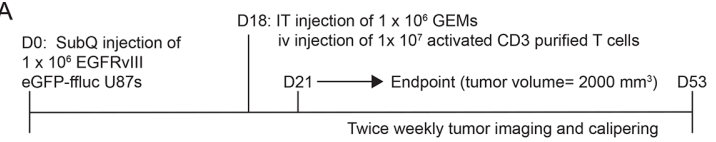

B
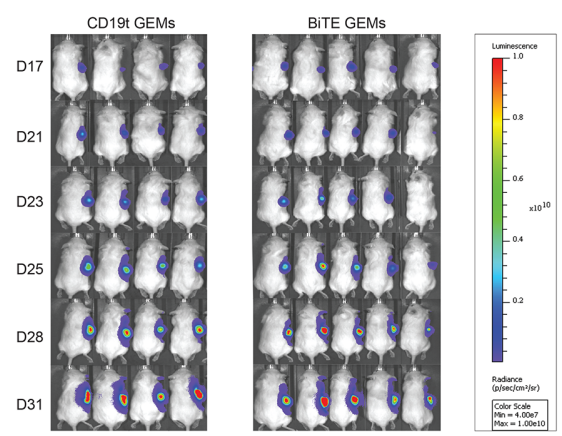

C

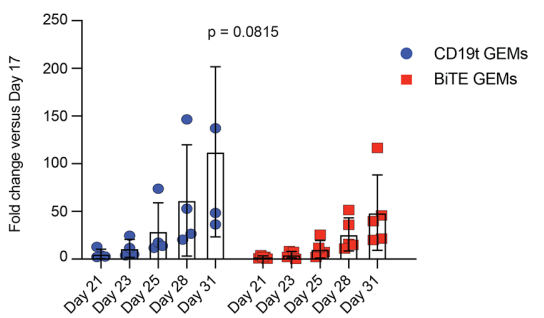

D

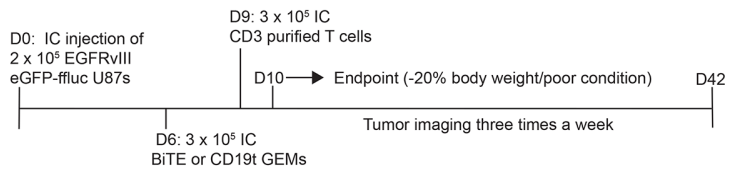

E
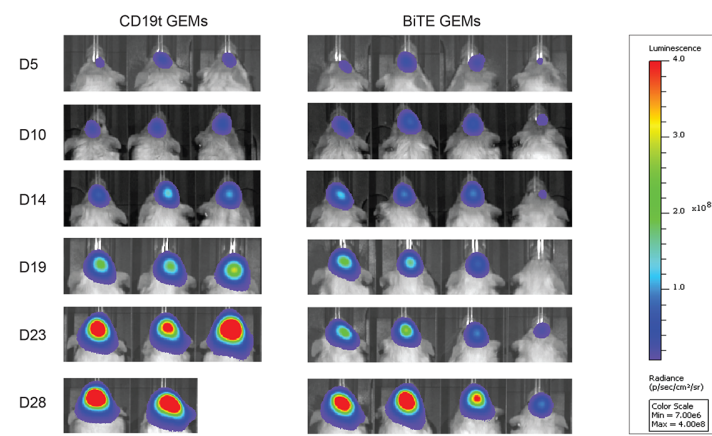

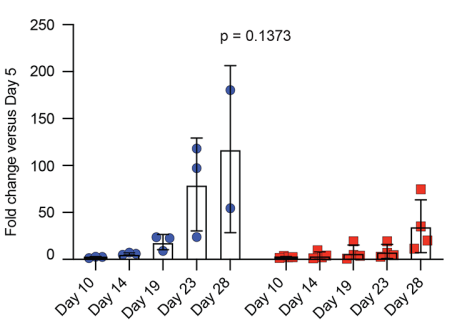

- CD19t GEMs

- BiTE GEMs

CD19t GEMs

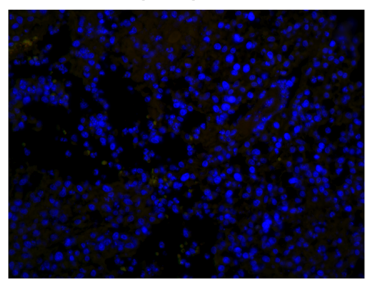

BiTE GEMs

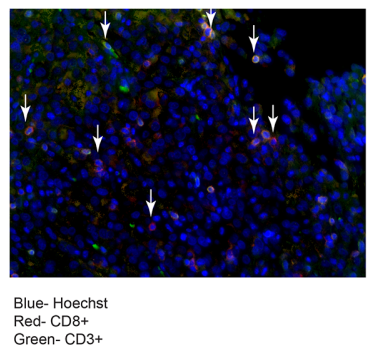

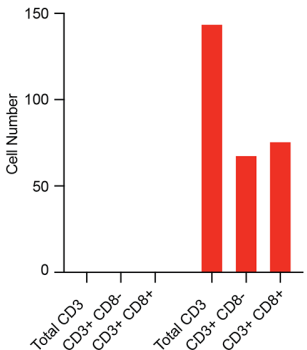

- CD19t GEMs - BiTE gems

Figure 5 Bispecific T cell engager (BiTE) genetically engineered macrophages (GEMs) reduce early tumor growth in in vivo mouse models of human glioblastoma and increase T cell infiltration to the tumor. (A) Scheme of in vivo subcutaneous experiment. Mice were injected subcutaneously with $1 \times 10^{6}$ epidermal growth factor receptor variant III (EGFRvIII) eGFP-ffluc U87s. On day 18 , mice received $1 \times 10^{6}$ BiTE or truncated CD19 (CD19t) control GEMs followed by intravenous injection of $1 \times 10^{7}$ activated T cells. (B) Luminescent images of mice that received CD19t control or BiTE GEMs in addition to activated T cells for time points listed to the left of images. Luminescent scale bar for imaging is shown to the right of images. (C) Fold change in average radians for each individual mouse in (B) from day 21 to 31 days post-U87 injection relative to day 17 is graphed. Values for individual mice in the CD19t GEM group are shown in blue circles and the BiTE GEM group are shown in red squares. Bars depict mean and SD for each mouse group. (D) Scheme of intracranial in vivo experiment. Mice were injected intracranially with $2 \times 10^{5}$ EGFRvIII eGFP-ffluc U87s. On day 6 following U87 injection, mice received $3 \times 10^{5} \mathrm{BiTE}$ GEMs or CD19t control GEMs intracranially. $3 \times 10^{5}$ purified CD3 T cells were injected 3 days following GEM injection. (E) Images displaying the intensity of luminescence for each mouse that received BiTE GEMs or CD19t control GEMs and T cells for time points as displayed on the left. Scale bar for imaging is shown to the right of the images. (F) Graph displays fold increase in luminescent signal for each individual mouse in BiTE (red squares) or CD19t (blue circles) GEM mouse group at indicated time points relative to day 5 post-U87 injection. Each bar represents mean signal per group and error bars depict SD. P values were determined for each time point using average fold changes for each time point and a paired t-test. (G) Tumors were harvested at the experimental endpoint (tumor volume $=2000 \mathrm{~mm}^{3}$ ) from mice that received subcutaneous tumors followed by IT GEMs and intravenous $\mathrm{T}$ cells as depicted in (A). Tumors were sectioned and stained for CD3 and CD8 and imaged at 40xon a Nuance microscope. A representative image is shown for each mouse group. The graph shows total CD3+ CD8-or CD3+ CD8+ Tcell numbers as quantified from 30 tumor sections from mice that received BiTE or CD19t control GEMs.

vivo differentiated and transduced GEMs. Gene ontogeny analysis shows that the genes activated in this setting are consistent with those observed after transduction with BiTE alone (online supplemental figure 6F).
In the flank U87 model, we found that the negative control, in which only $\mathrm{T}$ cells were injected, showed no delay in tumor growth, whereas the positive control testing $\mathrm{T}$ cells injected followed by intratumoral injection 


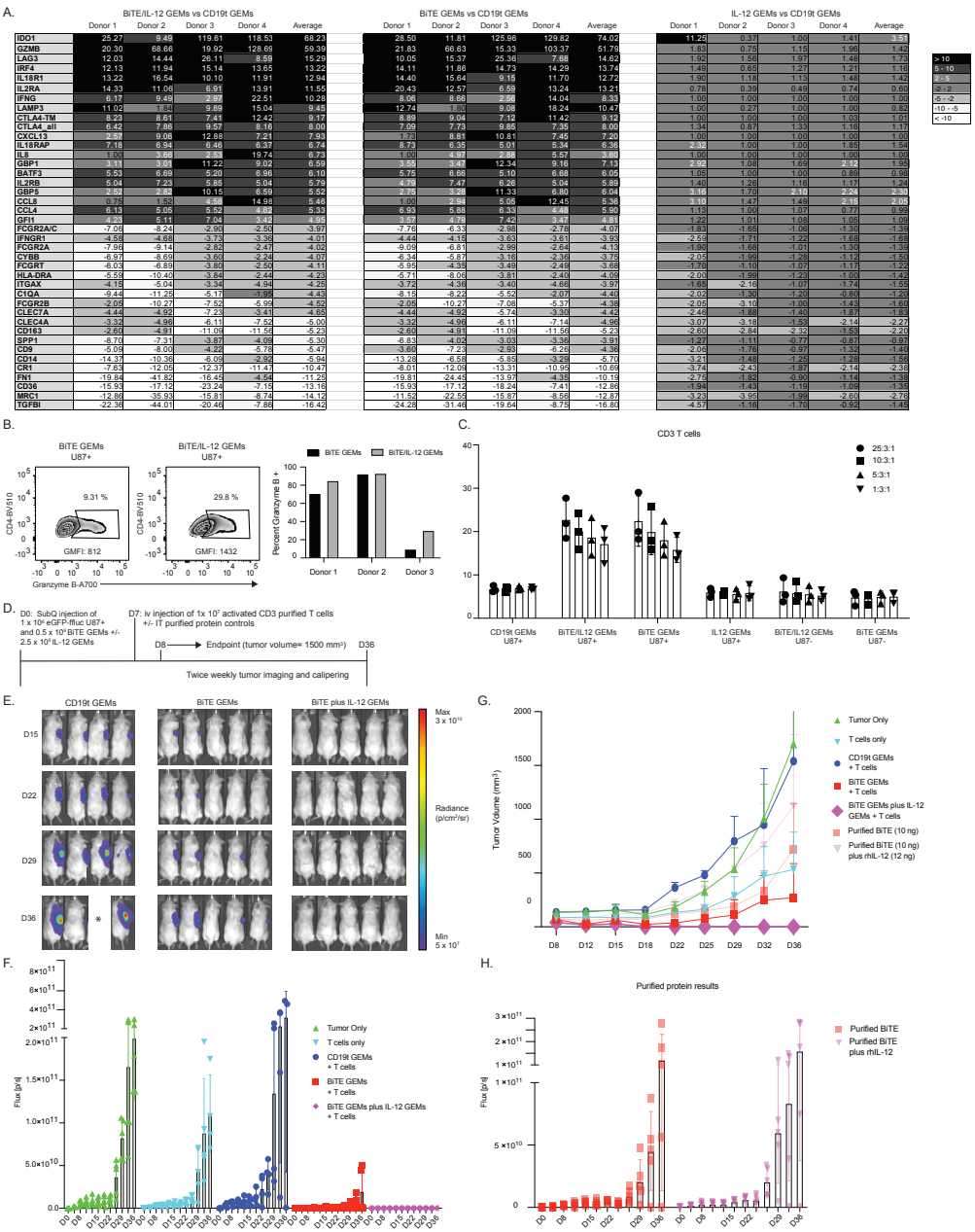

Figure 6 Interleukin (IL)-12 genetically engineered macrophages (GEMs) enhance T cell responses to bispecific T cell engager (BiTE) GEMs and prevent tumor growth in vivo in a subcutaneous glioblastoma model. (A) $3 \times 10^{6} \mathrm{~T}$ cells were cultured with $5 \times 10^{5} \mathrm{BiTE}$ or BiTE/IL-12 dual transduced GEMs and $2 \times 10^{5}$ epidermal growth factor receptor variant III (EGFRvIII) U87 target cells. On day 3 of culture, T cells were harvested, lysed, and RNA was isolated and run on a Nanostring human immunology v2 panel. The graphs show fold changes in normalized counts for the top 20 upregulated and downregulated genes for BiTE and IL-12 dual transduced GEMs, BiTE GEMs and IL-12 GEMs in comparison to cocultures with truncated CD19 (CD19t) control GEMs. (B) Representative dot plots of percentage of gated CD4 T cells positive for Granzyme B in the presence of BiTE (black) or BiTE/IL-12 (gray) dual transduced macrophages following a 3 day incubation of T cells, GEMs, and target cells at a ratio of 25:3:1. Results are shown for three independent donors in bar graphs to the right. (C) Purified CD3 T cells were incubated at specified ratios with BiTE/IL-12, BiTE, IL-12, or CD19t control GEMs in a 96-well plate. Chromium pulsed target cells were added for 18 hours following initial T cell and GEM incubation and percent lysis was determined based on chromium released into the supernatant during incubation relative to controls. Each symbol represents results from an independent donor with all ratios indicated run in triplicate. Ratios listed specify T cell:GEM:target cell. P values determined by t test for BiTE/ IL-12 versus C19t control GEM group were $0.004302,0.005281,0.010094$, and 0.012589 starting at the highest T cell ratio. Those for BiTE GEMs were 0.009650, 0.012134, 0.009186, and 0.006269. (D) Scheme for in vivo subcutaneous model. $1 \times 10^{6}$ EGFRvIII eGFP-ffluc U87s were injected subcutaneously in combination with $5 \times 10^{5}$ BiTE GEMs with or without $2.5 \times 10^{5}$ IL- 12 GEMs. Seven days following tumor establishment $1 \times 10^{7}$ Dynabead activated and rested T cells were injected intravenously. Control groups received $10 \mathrm{ng}$ purified BiTE protein with or without $12 \mathrm{ng}$ recombinant human IL-12 intratumorally. Mice were imaged twice weekly following T cell injection until experimental endpoint (tumor volume $=1500 \mathrm{~mm}^{3}$ or day 36 after tumor cell injection). (E) Luminescent images for individual mice from day 15, 22, 29, and 36 following tumor cell injection are shown from CD19t GEM, BiTE GEM, and BiTE plus IL-12 GEM mouse groups. Asterisk indicates mouse that was euthanized due to tumor volume. (F) Graph displays the luminescent signal for individual mice for the time points shown on the $x$-axis. The BiTE GEM group is shown in red and BiTE and IL-12 combination GEM group is shown in magenta. Each symbol represents an individual mouse. $\mathrm{p}<0.015$ for all time points list for BiTE plus IL-12 group compared with CD19t GEMs. For the day 36 time point for BiTE GEMs $p=0.044$. (G) Average tumor volume measurements are shown for the duration of the experiment for each of the mouse groups as determined by caliper measurements and indicated in the legend. Tumor volume $=\left(\right.$ width $^{2} \times$ length/2). $\mathrm{P}<0.02$ for all time points listed for the BiTE plus IL-12 GEM group in comparison to the T cell only group. (H) Luminescent signal for individual mice that received a single injection of intratumoral purified BiTE protein (10 ng, light red) or combination treatment of intratumoral purified BiTE protein (10 ng) and recombinant human IL-12 (12 ng), (light pink) is shown for the duration of the experiment. 
of purified BiTE protein and rhIL-12 resulted in complete resolution of subcutaneous tumor, indicating the potency of the combination (online supplemental figure $7 \mathrm{~A}, \mathrm{~B}$ ). To test effects on $\mathrm{T}$ cell functions in vitro, we performed gene expression analysis of T cells cultured with EGFRvIII target cells and either autologous BiTE GEMs or IL-12/ BITE GEMs. We found that dual transduced GEMs increased IFNG in comparison to BiTE GEMs alone (figure 6A), as well as a number of proinflammatory surface and secreted proteins (online supplemental figure 7C). Increased IFN $\gamma$ was not detected at the protein level in all donors (online supplemental figure $7 \mathrm{C}$ ), possibly due to rapid internalization by $\mathrm{T}$ cells and GEMs in the culture. Dual transduced cells significantly increased amounts of the proinflammatory proteins GMCSF and RANTES, and cytokines IL-6 and IL-8 (online supplemental figure 7C), which recruit and retain effector T cells to sites of inflammation. Consistent with $\mathrm{T}$ cell activation and proliferation shown using unbiased pathway analysis (online supplemental figure 6F), IL2RA was increased in all donors $(\mathrm{n}=4$, figure $6 \mathrm{~A})$ at the gene expression level, and IL-7 and IL-15 proteins were increased in supernatant (online supplemental figure 7C). IL-12 secretion also increased Granzyme B gene (figure 6A) and intracellular protein expression in CD4 T cells (figure 6B), although EGFRvIII targeted tumor cell cytotoxicity was dependent on GEM secretion of BiTE and was not significantly enhanced by the secretion of IL-12 (figure 6C).

To test the efficacy of BiTE/IL-12 produced by GEMs in vivo, GEMs were transduced with CD19t, BiTE alone, or both BiTE and IL-12, and injected into the flank U87 NSG tumor model (figure 6D). Intravenously injected $\mathrm{T}$ cells effectively prevented tumor growth for 36 days without any evidence of tumor (figure $6 \mathrm{E}-\mathrm{G}$ ), which was dependent on the transduction of GEMs with BiTE lentivirus, and enhanced with the dual production of BiTE and IL-12 (figure 6F). Intratumoral injection of purified BiTE or BiTE and IL-12 proteins delayed, but did not prevent tumor growth (figure 6G and $\mathrm{H}$ ). Collectively, our data suggest that the GEM platform may have added therapeutic benefit when used to deliver one or more therapeutic proteins locally.

\section{DISCUSSION}

In the current study, human macrophages were engineered to secrete a functional BiTE. BiTE secreted by GEMs activated T cells, induced cytokines and antigenspecific target cell lysis. With the novel demonstration of GEM lentivirally encoded scFv secretion, this platform may be used to produce therapeutic scFvs that may have targeting or toxicity barriers in a variety of indications.

GEMs as a therapeutic platform offer other advantages over current cellular therapies. Not unlike dendritic cells, macrophages express several proteins important for antigen presentation, and support of $\mathrm{T}$ cell effector functions. ${ }^{26}{ }^{27}$ This is supported here by the observation that GEMs induced more robust expression of activation genes and proteins than purified BiTE protein alone (figure 2). GEMs can also secrete a variety of therapeutic proteins, such as chemokines and cytokines, that could support proinflammatory immune responses in the TME. In contrast to CAR T or NK cells, macrophages do not proliferate,$^{28}$ thereby preventing delivery of potentially toxic concentrations of lentivirally encoded therapeutic proteins caused by rapid and unregulated proliferation of adoptively transferred cells. A particular strength of macrophages as a therapeutic cell for solid tumors includes their propensity for trafficking to and accumulation in solid tumors. ${ }^{29-31}$ Our previous work demonstrates the long-term survival, persistence of lentivirally encoded gene expression, and dissemination of locally injected GEMs throughout tumor tissue in vivo. ${ }^{11}$ In the current study, our data extend on initial characterization to show the potential for therapeutic efficacy when locally administered. Although this is an appealing avenue for tumors that are easily accessible for local delivery, an approach currently in clinical trials for other therapeutic platforms ${ }^{32}$ future studies will determine trafficking, recruitment, and efficacy of systemically injected GEMs for tumors that may be impossible to access, diffuse, multifocal, or metastatic.

The recent description of a macrophage engineered to express a human epidermal growth factor receptor 2-specific CAR capitalizes on the ability of macrophages to perform tumor-specific phagocytosis of tumor cells and expand the repertoire of peptides presented to $\mathrm{T}$ cells. ${ }^{2021}$ Our data indicate that GEMs could contribute to this interaction by both clearing debris resulting from tumor cell lysis and cross presenting novel tumor antigens to T cells (figure 3E).

GEMs can be transduced with at least two different lentiviruses (figure 6), so this platform allows testing of several therapeutic protein combinations. In addition to IL-12, which can increase the visibility of the tumor to the immune system, ${ }^{33}{ }^{34}$ costimulatory molecules or checkpoint inhibitors are also of interest. Although BiTE proteins do not require costimulation for $\mathrm{T}$ cell function, ${ }^{35}$ the addition of CD28 improves efficacy in mice. ${ }^{36-38}$ Increased PD-1 expression on T cells in solid tumors ${ }^{39}$ also suggests that $\mathrm{T}$ cell exhaustion can contribute to tumor outgrowth. Taken with studies exploring the mechanisms of checkpoint inhibition, ${ }^{40} 41$ and recent work showing that preventing $\mathrm{T}$ cell exhaustion promotes CAR $\mathrm{T}$ cell responses to solid tumors, ${ }^{42} 43$ the addition of costimulatory proteins or checkpoint blockade antibodies could therefore enhance BiTE efficacy.

This platform may also complement cellular immunotherapies, as indicated by recent work illustrating the impact of CAR T cells secreting BiTE protein in GBM. ${ }^{12}$ Although optimized therapeutic payloads delivered by GEMs may depend on indication, kinetics of immune response, and the type of companion cell therapy, it is appealing to consider this platform as an adjuvant to support the persistence, expansion, and functions of adoptively transferred cell therapies. 
Although the current study tested GEMs in the context of cancer, GEMs could be beneficial in other disease settings, such as viral infections and autoimmunity, where manipulation of local immune responses may improve patient outcomes. The use of a repertoire of lentiviral vectors to create indication-specific GEMs encoding immune modulatory proteins may therefore provide targeted combination therapies for a wide range of human immune diseases.

\section{Author affiliations}

${ }^{1}$ Ben Towne Center for Childhood Cancer Research, Seattle Children's Research Institute, Seattle, Washington, USA

${ }^{2}$ Department of Immunology, University of Colorado Denver School of Medicine, Aurora, Colorado, USA

${ }^{3}$ Division of Pediatric Neurosurgery, Seattle Children's Hospital, Seattle, Washington, USA

${ }^{4}$ Department of Neurological Surgery, University of Washington, Seattle, Washington, USA

${ }^{5}$ Ben Towne Center for Childhood Cancer, Seattle Children's Research Institute,

Seattle, Washington, USA

${ }^{6}$ Mozart Therapeutics, Seattle, WA, USE

Acknowledgements The authors thank Joseph Cheng and James Matthaei for thoughtful discussion and advice in these studies; Adam Johnson for assistance with scFv design; and the Jensen Lab for the gift of cell lines and technical training for intracranial in vivo studies.

Contributors JLG designed and performed studies, analyzed and interpreted data, generated figures, and prepared manuscript. LRM and HC assisted with manuscript and figure edits. LRM, KD, HC, and BP performed experiments, analyzed data, and supported mouse studies. SAK designed BiTE construct. SB performed western blots. AD generated human PBMCs used in all studies. CAC advised on study design and manuscript preparation. All authors read and approved final manuscript.

Funding This work was supported by Steven Higgins Brain Tumor Fund, the Aldarra Foundation, and Stand Up to Cancer.

Competing interests The technology used to generate genetically engineered macrophages in this manuscript is covered under patent \#US20170087185A1, Genetic engineering of macrophages for immunotherapy.

Patient consent for publication Not required.

Ethics approval Human blood products obtained commercially through Bloodworks Northwest. Mouse studies were conducted under Seattle Children's Research Institute IACUC standards (Protocol \# IACUC00088). Work with recombinant DNA was performed under IBC \#1211.

Provenance and peer review Not commissioned; externally peer reviewed.

Data availability statement All data relevant to the study are included in the article or uploaded as supplementary information. All data generated are included in this article and supplemental information.

Supplemental material This content has been supplied by the author(s). It has not been vetted by BMJ Publishing Group Limited (BMJ) and may not have been peer-reviewed. Any opinions or recommendations discussed are solely those of the author(s) and are not endorsed by BMJ. BMJ disclaims all liability and responsibility arising from any reliance placed on the content. Where the content includes any translated material, BMJ does not warrant the accuracy and reliability of the translations (including but not limited to local regulations, clinical guidelines, terminology, drug names and drug dosages), and is not responsible for any error and/or omissions arising from translation and adaptation or otherwise.

Open access This is an open access article distributed in accordance with the Creative Commons Attribution Non Commercial (CC BY-NC 4.0) license, which permits others to distribute, remix, adapt, build upon this work non-commercially, and license their derivative works on different terms, provided the original work is properly cited, appropriate credit is given, any changes made indicated, and the use is non-commercial. See http://creativecommons.org/licenses/by-nc/4.0/.

\section{ORCID iD}

Jennifer L Gardell http://orcid.org/0000-0002-2397-3468
REFERENCES

1 Bagley SJ, Desai AS, Linette GP, et al. Car T-cell therapy for glioblastoma: recent clinical advances and future challenges. Neuro Oncol 2018;20:1429-38.

2 Preusser M, Lim M, Hafler DA, et al. Prospects of immune checkpoint modulators in the treatment of glioblastoma. Nat Rev Neurol 2015;11:504-14.

3 Lawrence MS, Stojanov P, Polak P, et al. Mutational heterogeneity in cancer and the search for new cancer-associated genes. Nature 2013;499:214-8.

4 Choi BD, Gedeon PC, Herndon JE, et al. Human regulatory T cells kill tumor cells through granzyme-dependent cytotoxicity upon retargeting with a bispecific antibody. Cancer Immunol Res 2013;1:163-7.

5 Sayour EJ, McLendon P, McLendon R, et al. Increased proportion of Foxp3+ regulatory T cells in tumor infiltrating lymphocytes is associated with tumor recurrence and reduced survival in patients with glioblastoma. Cancer Immunol Immunother 2015;64:419-27.

6 Huehls AM, Coupet TA, Sentman CL. Bispecific T-cell engagers for cancer immunotherapy. Immunol Cell Biol 2015;93:290-6.

7 Choi BD, Kuan C-T, Cai M, et al. Systemic administration of a bispecific antibody targeting EGFRvIll successfully treats intracerebral glioma. Proc Natl Acad Sci U S A 2013;110:270-5.

8 Yang J, Yan J, Liu B. Targeting EGFRvIll for glioblastoma multiforme. Cancer Lett 2017;403:224-30.

9 Yu S, Li A, Liu Q, et al. Recent advances of bispecific antibodies in solid tumors. J Hematol Oncol 2017;10:155.

10 Strohl WR, Naso M. Bispecific T-Cell Redirection versus Chimeric Antigen Receptor (CAR)-T Cells as Approaches to Kill Cancer Cells. Antibodies 2019;8. doi:10.3390/antib8030041. [Epub ahead of print: $03 \mathrm{Jul} 2019]$.

11 Moyes KW, Lieberman NAP, Kreuser SA, et al. Genetically engineered macrophages: a potential platform for cancer immunotherapy. Hum Gene Ther 2017;28:200-15.

12 Choi BD, Yu X, Castano AP, et al. Car-T cells secreting bites circumvent antigen escape without detectable toxicity. Nat Biotechnol 2019;37:1049-58.

13 Wing A, Fajardo CA, Posey AD, et al. Improving CART-Cell therapy of solid tumors with oncolytic virus-driven production of a bispecific T-cell Engager. Cancer Immunol Res 2018;6:605-16.

14 Velasquez MP, Torres D, Iwahori K, et al. T cells expressing CD19specific Engager molecules for the immunotherapy of CD19-positive malignancies. Sci Rep 2016;6:27130.

15 Porter CE, Rosewell Shaw A, Jung Y, et al. Oncolytic adenovirus armed with bite, cytokine, and checkpoint inhibitor enables CAR $\mathrm{T}$ cells to control the growth of heterogeneous tumors. Mol Ther 2020;28:1251-62.

16 Speck T, Heidbuechel JPW, Veinalde R, et al. Targeted bite expression by an oncolytic vector augments therapeutic efficacy against solid tumors. Clin Cancer Res 2018;24:2128-37.

17 Kahlon KS, Brown C, Cooper LJN, et al. Specific recognition and killing of glioblastoma multiforme by interleukin 13-zetakine redirected cytolytic T cells. Cancer Res 2004;64:9160-6.

18 Berger C, Jensen MC, Lansdorp PM, et al. Adoptive transfer of effector CD8+ T cells derived from central memory cells establishes persistent T cell memory in primates. $J$ Clin Invest 2008;118:294-305.

19 Haryadi R, Ho S, Kok YJ, et al. Optimization of heavy chain and light chain signal peptides for high level expression of therapeutic antibodies in CHO cells. PLoS One 2015;10:e0116878.

20 Morrissey MA, Williamson AP, Steinbach AM, et al. Chimeric antigen receptors that trigger phagocytosis. Elife 2018;7. doi:10.7554/ eLife.36688. [Epub ahead of print: 04 Jun 2018].

21 Klichinsky M, Ruella M, Shestova O, et al. Human chimeric antigen receptor macrophages for cancer immunotherapy. Nat Biotechnol 2020;38:947-53.

22 Hao C, Parney IF, Roa WH, et al. Cytokine and cytokine receptor mRNA expression in human glioblastomas: evidence of Th1, Th2 and TH3 cytokine dysregulation. Acta Neuropathol 2002;103:171-8.

23 Compte M, Blanco B, Serrano F, et al. Inhibition of tumor growth in vivo by in situ secretion of bispecific anti-CEA $X$ anti-CD3 diabodies from lentivirally transduced human lymphocytes. Cancer Gene Ther 2007;14:380-8.

24 Steding CE, Wu S-tse, Zhang Y, et al. The role of interleukin-12 on modulating myeloid-derived suppressor cells, increasing overall survival and reducing metastasis. Immunology 2011;133:221-38.

25 Lieschke GJ, Rao PK, Gately MK, et al. Bioactive murine and human interleukin-12 fusion proteins which retain antitumor activity in vivo. Nat Biotechnol 1997;15:35-40. 
26 Asano K, Nabeyama A, Miyake Y, et al. CD169-positive macrophages dominate antitumor immunity by crosspresenting dead cellassociated antigens. Immunity 2011;34:85-95.

27 Backer R, Schwandt T, Greuter M, et al. Effective collaboration between marginal metallophilic macrophages and CD8+ dendritic cells in the generation of cytotoxic T cells. Proc Natl Acad Sci U S A 2010;107:216-21.

28 Metcalf $\mathrm{D}$. The molecular control of cell division, differentiation commitment and maturation in haemopoietic cells. Nature 1989;339:27-30.

29 Biswas SK, Allavena P, Mantovani A. Tumor-Associated macrophages: functional diversity, clinical significance, and open questions. Semin Immunopathol 2013;35:585-600.

30 Zhang Q-wen, Liu L, Gong C-yang, et al. Prognostic significance of tumor-associated macrophages in solid tumor: a meta-analysis of the literature. PLoS One 2012;7:e50946.

31 Medrek C, Pontén F, Jirström K, et al. The presence of tumor associated macrophages in tumor stroma as a prognostic marker for breast cancer patients. BMC Cancer 2012;12:306.

32 Algazi A, Bhatia S, Agarwala S, et al. Intratumoral delivery of tavokinogene telseplasmid yields systemic immune responses in metastatic melanoma patients. Ann Oncol 2020;31:532-40.

33 Tsung K, Meko JB, Peplinski GR, et al. II-12 induces T helper 1-directed antitumor response. J Immunol 1997;158:3359-65.

34 Gambotto A, Tüting T, McVey DL, et al. Induction of antitumor immunity by direct intratumoral injection of a recombinant adenovirus vector expressing interleukin-12. Cancer Gene Ther 1999;6:45-53.
35 Dreier T, Lorenczewski G, Brandl C, et al. Extremely potent, rapid and costimulation-independent cytotoxic T-cell response against lymphoma cells catalyzed by a single-chain bispecific antibody. Int $J$ Cancer 2002;100:690-7.

36 Velasquez MP, Szoor A, Vaidya A, et al. Cd28 and 41BB costimulation enhances the effector function of CD19-specific Engager T cells. Cancer Immunol Res 2017;5:860-70.

37 Correnti CE, Laszlo GS, de van der Schueren WJ, et al. Simultaneous multiple interaction T-cell engaging (SMITE) bispecific antibodies overcome bispecific T-cell engager (bite) resistance via CD28 costimulation. Leukemia 2018;32:1239-43.

38 Laszlo GS, Gudgeon CJ, Harrington $\mathrm{KH}$, et al. T-Cell ligands modulate the cytolytic activity of the CD33/CD3 bite antibody construct, AMG 330. Blood Cancer J 2015;5:e340.

39 Blank C, Mackensen A. Contribution of the PD-L1/PD-1 pathway to T-cell exhaustion: an update on implications for chronic infections and tumor evasion. Cancer Immunol Immunother 2007;56:739-45.

40 Wei SC, Duffy CR, Allison JP. Fundamental mechanisms of immune checkpoint blockade therapy. Cancer Discov 2018;8:1069-86.

41 Ribas A, Wolchok JD. Cancer immunotherapy using checkpoint blockade. Science 2018;359:1350-5.

42 Cherkassky L, Morello A, Villena-Vargas J, et al. Human CAR T cells with cell-intrinsic PD-1 checkpoint blockade resist tumor-mediated inhibition. J Clin Invest 2016;126:3130-44.

43 Song Y, Liu Q, Zuo T, et al. Combined antitumor effects of anti-EGFR variant III CAR-T cell therapy and PD-1 checkpoint blockade on glioblastoma in mouse model. Cell Immunol 2020;352:104112. 